\title{
Nucleon Form Factors
}

\section{R. Alarcon ${ }^{1}$}

Department of Physics, Arizona State University

Tempe, AZ, 85287-1504, USA

E-mail: ralarconeasu.edu

\begin{abstract}
Measurements of the electric and magnetic form factors have been carried out by means of scattering of polarized electrons from vector-polarized hydrogen and deuterium. The experiment used the longitudinally polarized stored electron beam of the MIT-Bates South Hall Ring along with an isotopically pure, highly vector-polarized internal atomic hydrogen and deuterium target provided by an atomic beam source, and the symmetric Bates Large Acceptance Spectrometer Toroid (BLAST) with enhanced neutron detection capability. The measurements are at low momentum transfers $\left(\mathrm{Q}^{2}<1(\mathrm{Gev} / \mathrm{c})^{2}\right)$ and results are presented for the proton form factor ratio $G_{E}{ }^{p} / G_{M}{ }^{p}$ and for the electric form factor of the neutron, $G_{E}{ }^{n}$. To quantify the effect of multiple photon exchange and to address the puzzle of the proton form factor ratio at $\mathrm{Q}^{2}>1(\mathrm{GeV} / \mathrm{c})^{2}$, an experiment under development using BLAST and an unpolarized internal hydrogen target will measure electron-proton and positron-proton elastic scattering.
\end{abstract}

6th International Workshop on Chiral Dynamics - CD09

Bern, Switzerland,

July 6-10 2009

\footnotetext{
${ }^{1}$ Speaker
} 


\section{Introduction}

The electromagnetic structure of the nucleon is traditionally described in terms of two form factors, and it has been extensively studied by the scattering of electrons on nucleons [1]. Experiments on electron-proton scattering have yielded abundant information on the magnetic $G_{M}{ }^{p}$ and charge $G_{E}{ }^{p}$ form factors of the proton in the range of momentum transfers squared, $\mathrm{Q}^{2}$, up to about $30(\mathrm{GeV} / \mathrm{c})^{2}$. The information on the neutron form factors comes almost entirely from the scattering of electrons on deuterons or ${ }^{3} \mathrm{He}$ nuclei. In recent years, the combination of polarized electron beams with polarized targets or with recoil polarimeters has made possible new classes of experiments aimed at extracting the nucleon form factors utilizing spin degrees of freedom.

Measurements of the nucleon form factors present a sensitive test of nucleon models and QCD-inspired theories. In addition, accurate measurements of the form factors at low $\mathrm{Q}^{2}$ are required to reduce systematic uncertainty in the extraction of the strange quark contribution to the nucleon electromagnetic structure as studied in parity-violation electron scattering experiments [2].

This work presents nucleon form factors results obtained from the scattering of polarized electrons on polarized internal gas targets of hydrogen and deuterium using a large acceptance detector (BLAST) at the South Hall Ring of the MIT-Bates Linear Accelerator Center. The measurements span the low- $\mathrm{Q}^{2}$ range between 0.1 and $0.8(\mathrm{GeV} / \mathrm{c})^{2}$.

The paper is organized as follows: Section 2 describes briefly the BLAST experiment. Section 3 presents the BLAST results obtained for the proton form ratio $G_{E}{ }^{p} / G_{M}{ }^{p}$. Also shown are proyections of the OLYMPUS experiment for the ratio of positron-proton to electron-proton elastic scattering, with the goal of quantifying the effect of the multiple photon exchange in lepton scattering. These measurements intend to address the $G_{E}{ }^{p} / G_{M}{ }^{p}$ discrepancy at $\mathrm{Q}^{2}>1$ $(\mathrm{GeV} / \mathrm{c})^{2}$ between polarization experiments and cross section measurements. Section 4 presents the results obtained for the electric form factor of the neutron with BLAST in conjunction with the world data for this important observable. A summary is presented in Section 5.

\section{The BLAST Experiment}

At the MIT-Bates Linear Accelerator Center, the nucleon form factors have been measured by scattering polarized electrons from vector-polarized hydrogen and deuterium. The experiment used the longitudinally polarized electron beam stored in the MIT-Bates South Hall Ring along with an isotopically pure, highly vector-polarized internal atomic hydrogen and deuterium target provided by an atomic beam source. The measurements were carried out with the symmetric Bates Large Acceptance Spectrometer Toroid (BLAST) [3].

The BLAST detector was built as a toroidal spectrometer consisting of eight normalconducting copper coils producing a maximum field of $3800 \mathrm{G}$. The two in-plane sectors opposing each other were symmetrically equipped with drift chambers for the reconstruction of charged tracks, aerogel-Cerenkov detectors for $e-\pi$ discrimination and $1 "$ thick plastic 
scintillators for timing, triggering and particle identification. The angular acceptance covered scattering angles between $20^{\circ}$ and $80^{\circ}$ and $\pm 15^{\circ}$ out of plane. The symmetric detector core was surrounded by thick large-area walls of plastic scintillators for the detection of neutrons using the time-of-flight method.

The BLAST experiment made use of elastic ep-scattering of polarized electrons from polarized hydrogen to access the proton form factors, quasielastic $\left(\boldsymbol{e}, e^{\prime} n\right)$ scattering from

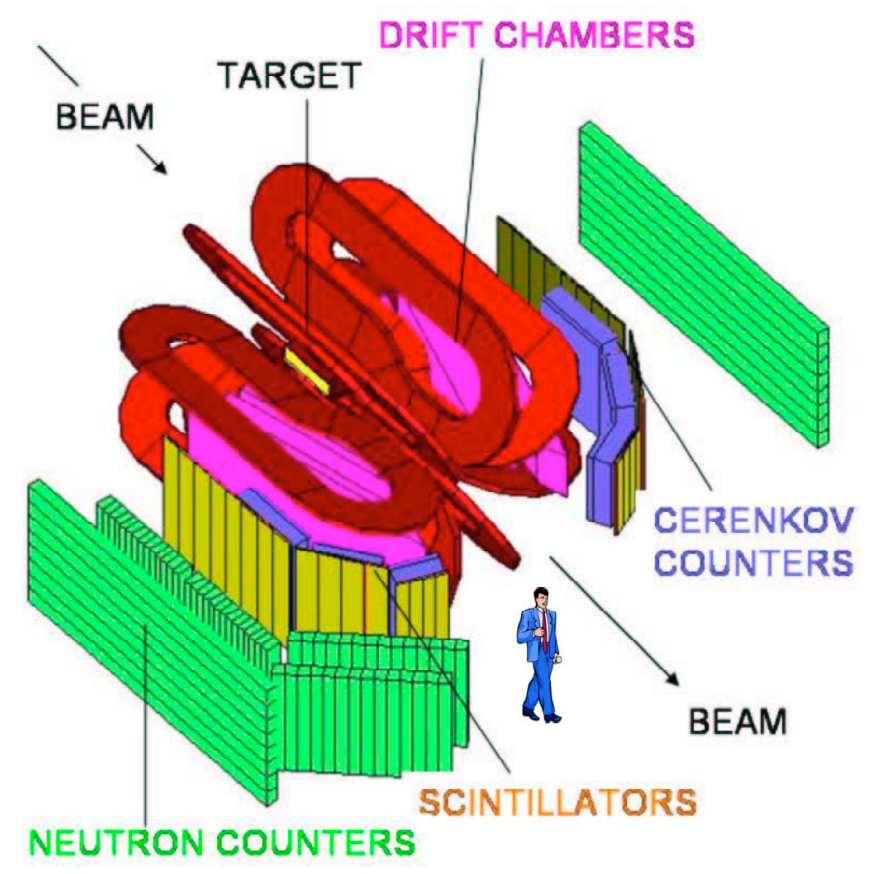

Figure 1: Schematic, isometric view of the BLAST detector showing the main detector elements.

vector-polarized deuterium to get at the charge form factor of the neutron, inclusive $\left(\boldsymbol{e}, e^{\prime}\right)$ scattering from vector-polarized deuterium for the magnetic form factor of the neutron, and elastic $e d$-scattering to measure tensor analyzing powers to help separate the deuteron form factors.

\section{The Proton Form Factors}

The BLAST results for the proton form factor ratio $G_{E}{ }^{p} / G_{M}{ }^{p}$ [4] are shown in Fig. 2 together with published recoil polarization [5-11], together with a few selected models: a soliton model [12], an extended vector meson dominance model [13], an updated dispersion model [14], a relativistic constituent quark model (CQM) with SU(6) symmetry breaking and a constituent quark form factor [15], and a Lorentz covariant chiral quark model [16]. The BLAST polarized hydrogen data were divided into eight $\mathrm{Q}^{2}$ bins and the yield distributions were in good agreement with results from a Monte Carlo simulation, including all detector efficiencies measured from the data. The BLAST detector configuration was symmetric about 
the incident electron beam and the target polarization angle was oriented $\sim 45^{0}$ to the left of the beam. Two independent asymmetries of electrons scattered into the beam-left and beam-right sectors, respectively, were measured simultaneously. The ratio $G_{E}{ }^{p} / G_{M}{ }^{p}$ could then be determined, independent of the polarization of the beam and the target, from the ratio of these experimental asymmetries measured at the same $\mathrm{Q}^{2}$ value but corresponding to different spin orientations [17].

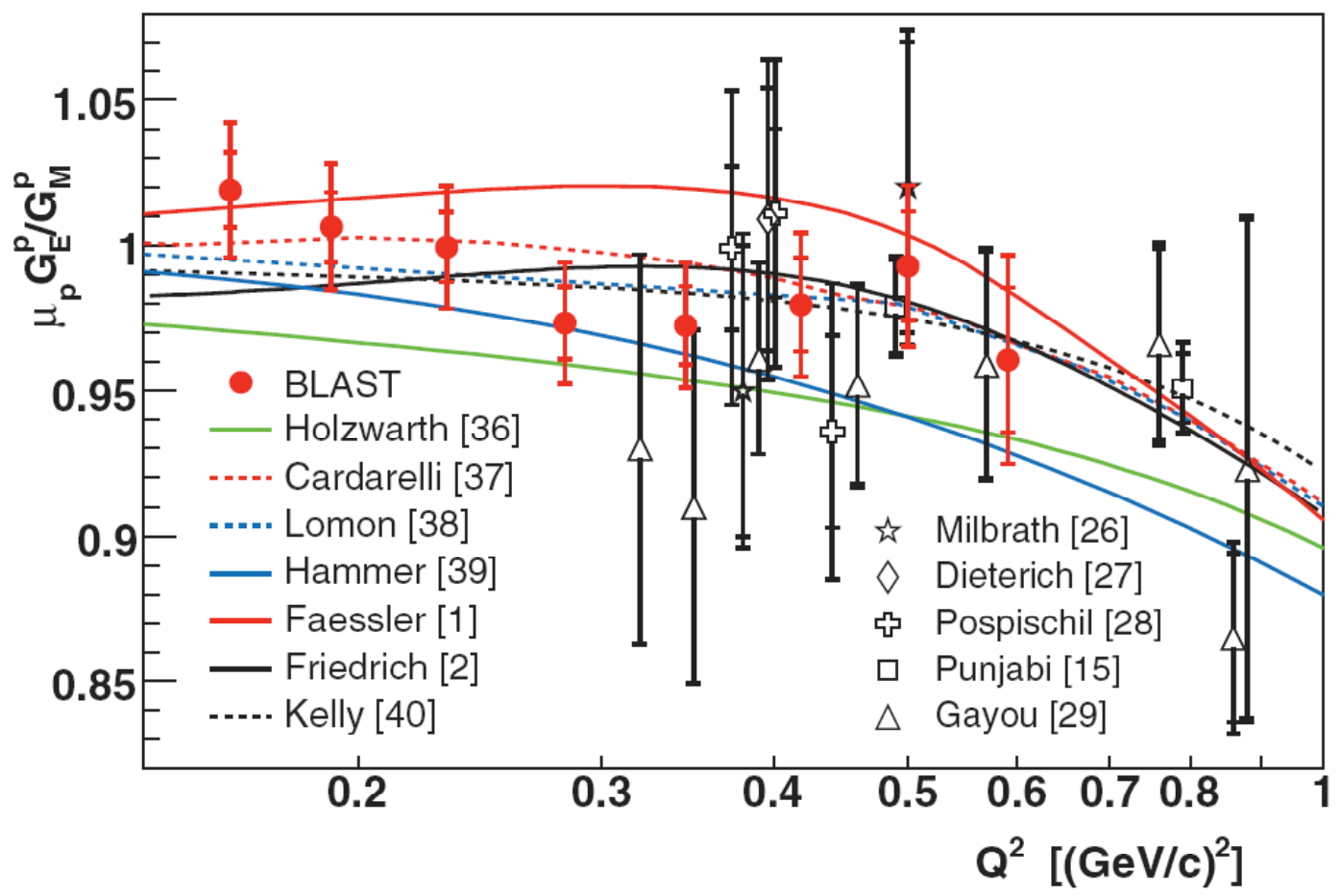

Figure 2: Results from BLAST for the proton form factor ratio shown with previous polarization data as a function of $\mathrm{Q}^{2}$ up to $1(\mathrm{GeV} / \mathrm{c})^{2}$. A new recoil polarization measurement has been published [18] in this $\mathrm{Q}^{2}$ range that is consistent with the BLAST results.

The BLAST experimental technique is complementary to the recoil polarization technique, in which the polarization of the recoil nucleon is measured instead of using a polarized target. For example, using the technique of recoil polarization transfer it is possible to measure directly the proton form factor ratio, $G_{E}^{p} / G_{M}^{p}[5]$.

Measurements of $G_{E}^{p} / G_{M}{ }^{p}$ have been carried out at JLab using this polarization technique and a continuous, highly polarized electron beam. The results have revealed a linear drop in the $G_{E}^{p} / G_{M}^{p}$ ratio up to the largest available value of $\mathrm{Q}^{2} \approx 6(\mathrm{GeV} / \mathrm{c})^{2}$ [7]. Surprisingly, the drop was actually in agreement with old phenomenological models based on vector meson dominance dispersion relations [19]. New fits using these parameterizations confirmed the dramatic drop with the astonishing prediction that the proton charge form factor $G_{E}{ }^{p}$ would vanish at $\mathrm{Q}^{2} \approx 8$ $(\mathrm{GeV} / \mathrm{c})^{2}$ and then it would change sign [20].

The $G_{E}^{p} / G_{M}{ }^{p}$ polarization transfer measurements at $\mathrm{Q}^{2}>1(\mathrm{GeV} / \mathrm{c})^{2}$ do not agree with the form factor ratio obtained using the traditional Rosenbluth separation technique [21] with cross 
section measurements. At first the disagreement was thought to be of an experimental nature. The Rosenbluth data were older and taken at low duty-cycle electron accelerators, and statistics did not match that obtained at the continuous electron beam facility of JLab. In an attempt to resolve the impasse, new measurements using the Rosenbluth technique were carried out at JLab now using the same beam as the polarization transfer measurements and high-resolution spectrometers available at JLab. These new Rosenbluth results [22] were in excellent agreement

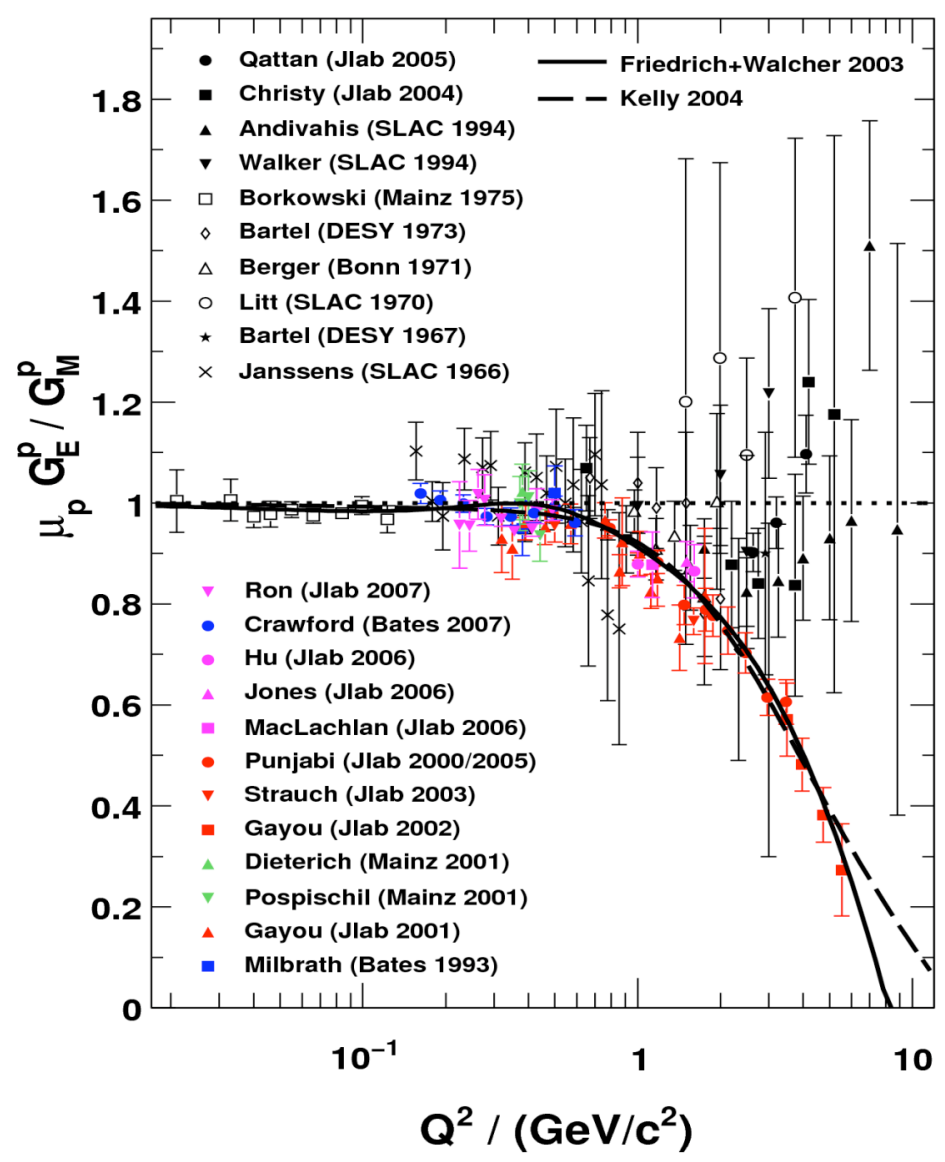

Figure 3: (Left): World data for the ratio of proton electric to magnetic form factor as a function of $\mathrm{Q}^{2}$.

with previous Rosenbluth data and showed a dramatic discrepancy with the polarization transfer results. This is the puzzle of the proton form factor ratio. The data are summarized in Fig. 3.

This puzzle has been explained as the effects of multiple photon exchange beyond the usual one-photon exchange approximation in the calculation of the elastic electron-proton scattering cross section [23]. It is therefore essential to definitively verify the contribution of multiple photon exchange since most of our understanding on the structure of the proton and atomic nuclei is based upon lepton scattering analyzed in terms of the single photon approximation. The most direct evidence for multiple photon exchange would be a deviation from unity in the ratio of positron-proton to electron-proton elastic scattering cross sections. 
To this end an experiment (OLYMPUS) has been proposed [24] that would utilize intense beams of electrons and positrons to precisely measure elastic scattering with high statistical and systematic precision. The OLYMPUS experiment will measure the ratio of electron-proton to positron-proton elastic cross sections with the BLAST detector using an internal, unpolarized hydrogen target and intense stored beams of unpolarized positrons and electrons at an energy of $2.0 \mathrm{GeV}$ at the site of the ARGUS experiment on the storage ring DORIS at DESY in Germany.

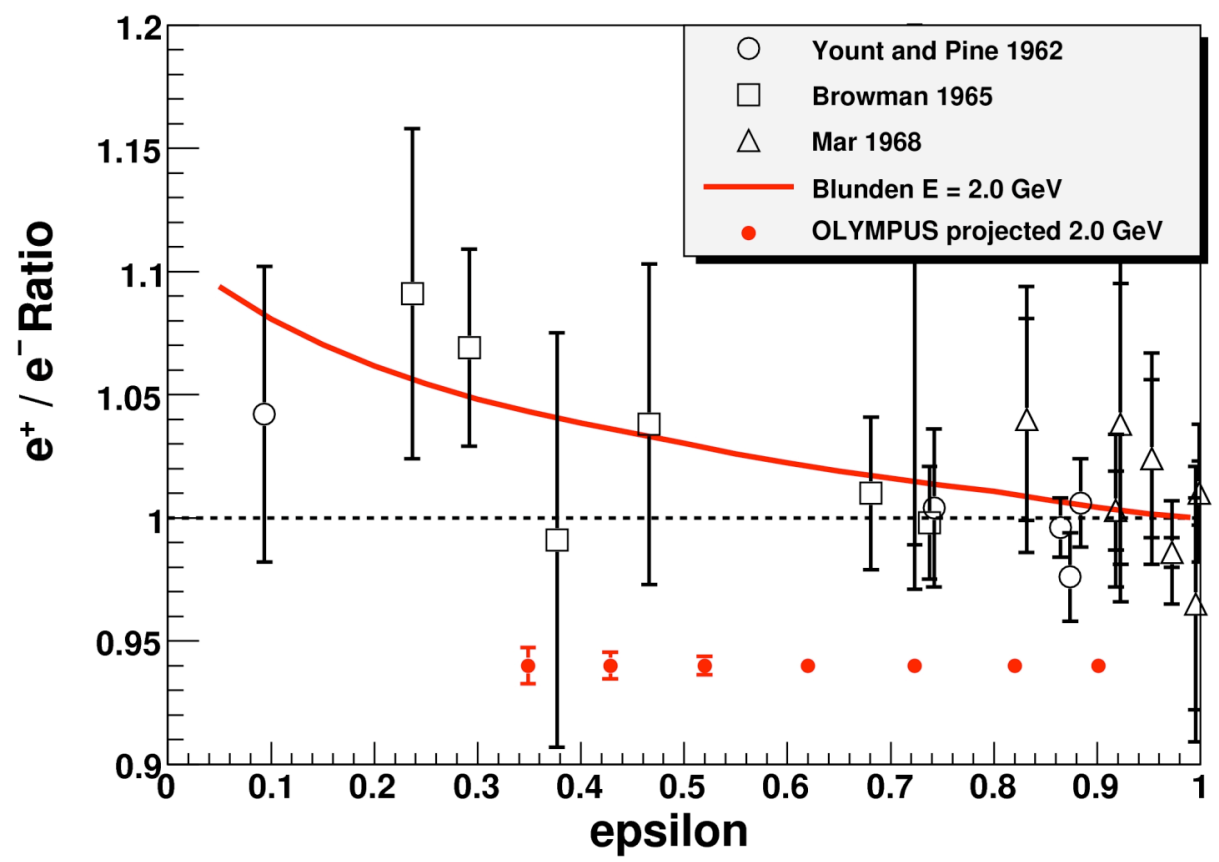

Figure 4: The ratio of the $e^{+}-p$ to $e^{-}-p$ cross sections as a function of the virtual photon polarization for the kinematics of the OLYMPUS measurement. The open symbols are the present data, the solid red points the OLYMPUS projections, and the curve is an example of the many theoretical models presently used to calculate the ratio.

To carry out this experiment it will be required to operate the DORIS storage ring at an energy of $2.0 \mathrm{GeV}$, to switch between beams of electrons and positrons at a frequency on the order of once per day, to relocate the BLAST detector from MIT-Bates to DESY/DORIS, and to install an unpolarized hydrogen internal gas target in the DORIS storage ring. Measurements from BLAST will allow a determination of the cross section ratio as a function of the virtual photon polarization $\varepsilon$, and hence the size of the multiple photon contribution. Fig. 4 shows the projected uncertainties of the positron-electron cross section ratio as a function of $\varepsilon$, assuming a luminosity of $2 \times 10^{33} \mathrm{~cm}^{2} \mathrm{~s}^{-1}$ and a running time of $500 \mathrm{~h}$ for both $\mathrm{e}^{+}$and $\mathrm{e}^{-}$. Also shown is a theoretical prediction [25] for the cross section ratio, evaluated for constant beam energy of 2.0 $\mathrm{GeV}$ as a function of $\varepsilon$. 


\section{The Neutron Form Factors}

The electric form factor of the neutron, $G_{E}{ }^{n}$, is an ideal testing ground for QCD- and pioncloud inspired and other effective nucleon models. A precise knowledge of $G_{E}{ }^{n}$ at low $\mathrm{Q}^{2}$ is also essential to reduce the systematic errors of parity violation experiments [2].

With BLAST measurements of $G_{E}{ }^{n}$ were carried out by means of $\left(\boldsymbol{e}, e^{\prime} n\right)$ quasi-elastic scattering using polarized electrons and a vector-polarized deuterium target. The experimental double spin asymmetry was formed from the measured (e,e'n)-yields in each beam-target spin state combination, properly normalized to the collected deadtime-corrected beam charge. For five bins in $\mathrm{Q}^{2}$, the experimental asymmetry as a function of missing momentum was compared with the full BLAST Montecarlo result based on deuteron electrodisintegration cross section calculations with consistent inclusion of reaction mechanism and deuteron structure effects. The electric form factor of the neutron was varied as an input parameter to the Montecarlo simulation and its measured value was extracted by a chi-squared minimization for each $\mathrm{Q}^{2}$ bin [25-26].

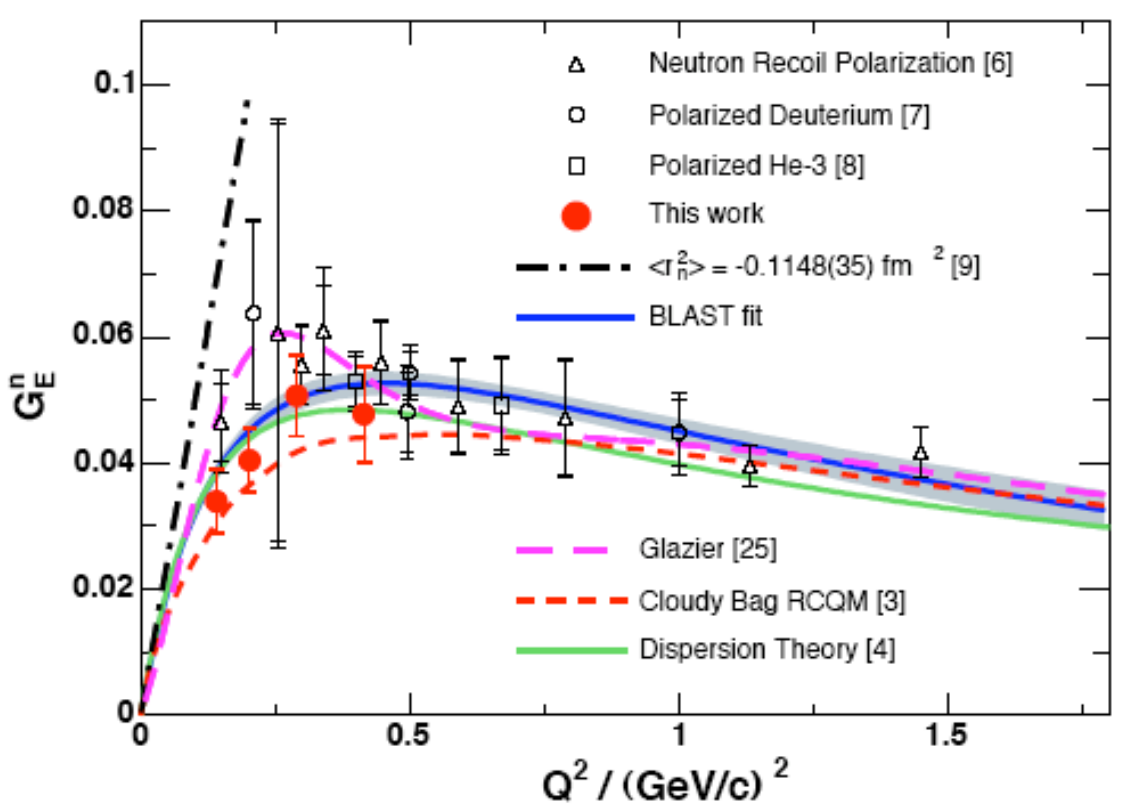

Figure 5: Elastic form factor of the neutron, $G_{E}{ }^{n}$, from polarization experiments along with results from BLAST.

The BLAST results [27] are displayed in Fig. 5 along with the world's data on the neutron charge form factor $\mathrm{G}_{\mathrm{E}}{ }^{\mathrm{n}}$ from double polarization experiments [28]. The BLAST data are in excellent agreement with the dispersion analysis [29] and also agree well with the meson-cloud model [30].

Measurements of the magnetic form factor of neutron $G_{M}{ }^{n}$ have also exploited the recent advances in polarization techniques. In particular, the inclusive quasi-elastic reaction, ${ }^{3} \mathrm{He}\left(\boldsymbol{e}, e^{\text {') }}\right.$, exploits the fact that in the ground state of ${ }^{3} \mathrm{He}$ the proton spins anti-align and cancel and the 
spin of the nucleus is carried by the unpaired neutron [31]. By the simultaneous use of beam and target polarization the experimental asymmetry is related to the ratio of the form factors of the neutron [32]. The systematic uncertainties for these measurements stem mainly from final state interactions (FSI) and reaction mechanisms like meson exchange currents (MEC) and isobar configurations (IC) corrections.

With BLAST an alternative measurement of $G_{M}{ }^{n}$ has been carried out by using inclusive electron scattering with polarized beam and vector-polarized deuterium target, ${ }^{2} \boldsymbol{H}\left(\boldsymbol{e}, e^{\prime}\right)$, in quasi-elastic kinematics. The two-body nature of the deuteron allows analytic solutions to its nuclear structure. Additionally, in the quasi-elastic regime, the FSI, MEC and IC are fairly small. These data are under final analysis and publication of final results is forthcoming.

\section{Summary}

Nucleon form factors results were presented from the scattering of polarized electrons on polarized internal gas targets of hydrogen and deuterium using the BLAST detector at the South Hall Ring of the MIT-Bates Linear Accelerator Center. The measurements span the low- $\mathrm{Q}^{2}$ range between 0.1 and $0.8(\mathrm{GeV} / \mathrm{c})^{2}$. The results for the $G_{E}{ }^{p} / G_{M}{ }^{p}$ ratio and for $G_{E}{ }^{n}$ are more precise than previous data in the corresponding $\mathrm{Q}^{2}$ range.

The world results for $G_{E}{ }^{n}$ from polarization experiments show a remarkable consistency and have conduced to the precise determination of this important observable up to about 1.5 $(\mathrm{GeV} / \mathrm{c})^{2}$. New measurements are underway and/or planned at JLab to determine $G_{E}{ }^{n}$ at higher values of $\mathrm{Q}^{2}$.

The $G_{E}{ }^{p} / G_{M}{ }^{p}$ polarization transfer measurements at $\mathrm{Q}^{2}>1(\mathrm{GeV} / \mathrm{c})^{2}$ do not agree with the form factor ratio obtained from cross section measurements. Projections were shown for the OLYMPUS experiment which is under development and will utilize BLAST and an unpolarized internal hydrogen target. The goal of OLYMPUS is to measure electron-proton and positronproton elastic scattering in order to quantify the effect of multiple photon exchange and thus address the above disagreement.

\section{References}

[1] P. Bosted, Phys. Rev. C 51, 409 (1995); J.J. Kelly, Phys. Rev. C 70, 068202 (2004).

[2] D.S. Armstrong et al., Phys. Rev. Lett. 95, 092001 (2005).

[3] D. Hasell et al., Nucl. Instr. Meth. A 603, 247-262 (2009).

[4] C. B. Crawford et al, Phys. Rev. Lett. 98, 52301 (2007).

[5] M. Jones et al., Phys. Rev. Lett. 84, 1398 (2000).

[6] O. Gayou et al., Phys. Rev. Lett. 88, 092301 (2002).

[7] V. Punjabi et al., Phys. Rev. C 71, 055202 (2005).

[8] O. Gayou et al., Phys. Rev. C 64, 038202 (2001). 
[9] S. Dieterich et al., Phys. Lett. B 500, 47 (2001).

[10] T. Pospischil et al., Eur. Phys. J. A 12, 125 (2001).

[11] B. Milbrath et al., Phys. Rev. Lett. 80, 452 (1998), and 82, 2221(E) (1999).

[12] G. Holzwarth, Z. Phys. A 356, 339 (1996).

[13] E.L. Lomon, Phys. Rev. C 66, 045501 (2002).

[14] H.-W. Hammer and Ulf-G. Meissner, Eur. Phys. J. A 20, 469 (2004).

[15] F. Cardarelli and S. Simula, Phys. Rev. C 62, 065201 (2000).

[16] A. Faessler, T. Gutsche, V. Lyubovitskij, K. Pumsaard, Phys. Rev. D 73, 114021 (2006).

[17] C.B. Crawford, Ph.D. thesis, Massachusetts Institute of Technology (2005).

[18] G. Ron et al., Phys. Rev. Lett. 99, 202002 (2007).

[19] F. Iachello, A. D. Jackson, and A. Lande, Phys. Lett. B 43, 191 (1973).

[20] R. Bijker and F. Iachello, Phys. Rev. C 69, 068201 (2004).

[21] M. N. Rosenbluth, Phys. Rev. 79, 615 (1950).

[22] I. A. Qattan et al., Phys. Rev. Lett. 94, 142301 (2005).

[23] S. Kondratyuk, P. Blunden, W. Melnitchouk, and T. A. Tjon, Phys. Rev. Lett. 95, 172503 (2005); Yu. M. Bystritskiy, E. A. Kuraev, and E. Tomasi-Gustafsson, Phys. Rev. C 75, 015207 (2007); and references therein.

[24] "A Proposal to Definitively Determine the Contribution of Multiple Photon Exchange in Elastic Lepton-Nucleon Scattering”, The OLYMPUS Collaboration, DESY, September 9, 2008.

[25] V. Ziskin, Ph.D. Thesis, Massachusetts Institute of Technology (2005).

[26] E. Geis, Ph.D. Thesis, Arizona State University (2007).

[27] E. Geis, M. Kohl, V. Ziskin, et al., Phys. Rev. Lett. 101, 042501 (2008).

[28] D.I. Glazier et al., Eur. Phys. J. A 24, 101 (2005) and references therein.

[29] M.A. Belushkin, H.-W. Hammer, and U.-G. Meissner, Phys. Rev. C 75, 035202 (2007).

[30] G.A. Miller, Phys. Rev. C 66, 032201 (2002).

[31] J.L. Friar et al., Phys. Rev. C 42, 2310 (1990).

[32] H. Gao et al., Phys. Rev. C 50, R546 (1994); W. Xu et al., Phys. Rev. Lett. 85, 2900 (2000); W. Xu et al., Phys. Rev. C 67, R012201 (2003). 